Published in final edited form as:

Leuk Lymphoma. 2016 June ; 57(6): 1423-1428. doi:10.3109/10428194.2015.1091928.

\title{
A prospective analysis of blood donation history and risk of non-Hodgkin lymphoma
}

\author{
Kazusa Ishii ${ }^{1}$, Brenda M. Birmann ${ }^{2}$, Xuehong Zhang $^{2}$, Edward Giovannucci ${ }^{2,3,4}$, and \\ Kimberly A. Bertrand ${ }^{2,3}$ \\ ${ }^{1}$ Hematology Branch, National Heart, Lung, and Blood Institute, National Institutes of Health, \\ Bethesda, MD 20892 \\ ${ }^{2}$ Channing Division of Network Medicine, Department of Medicine, Brigham and Women's \\ Hospital and Harvard Medical School, Boston, MA 02115 \\ ${ }^{3}$ Department of Epidemiology, Harvard T.H. Chan School of Public Health, Boston, MA 02115 \\ ${ }^{4}$ Department of Nutrition, Harvard T.H. Chan School of Public Health, Boston, MA 02115
}

\begin{abstract}
Blood donation may influence subsequent NHL development via temporary immune system alterations. To test the hypothesis that frequent blood donation is associated with an increased risk of NHL and its most common histologic subtypes, we followed 36,576 men in the Health Professionals Follow-up Study (HPFS), who provided information on frequency of blood donation in the past 30 years in 1992. We confirmed 544 incident cases of NHL through 2010. Cox proportional hazards regression was used to calculate hazards ratios (HR) and 95\% CI for the risk of all NHL and major NHL histologic subtypes associated with number of blood donations. In this prospective study, there was no significant evidence of an association between blood donation frequency and incidence of NHL (age-adjusted HR 1.26, 95\% CI 0.94-1.68, comparing >20 donations vs. 0 donations over 30 years, $\mathrm{p}$ for trend $=0.18$ ) or of any major NHL subtypes.
\end{abstract}

\section{Keywords}

blood donation; non-Hodgkin lymphoma; epidemiology; risk

\section{INTRODUCTION}

Non-Hodgkin lymphoma (NHL) is a heterogeneous group of lymphoid malignancies [1]. Incidence rates of NHL have markedly increased over the last few decades worldwide, but the reason for such increase has been largely unexplained [1,2]. Among known risk factors, severe immune deficiency is well established to increase the risk of NHL [2]. Immunemodulating stimuli and exposures are also associated with the risk of NHL [3-5].

Corresponding author: Kimberly A. Bertrand, Channing Division of Network Medicine, Brigham and Women's Hospital \& Harvard Medical School, 181 Longwood Avenue, Boston, MA 02115, phone: (617) 525-2140, fax: (617) 525-2008,

kbertran@hsph.harvard.edu.

Declaration of interests: The authors declare no competing financial interests. 
Blood donation has been demonstrated to cause temporary immune system alterations in human studies [6-8]. It is not yet known whether such temporary immune system alterations following blood donation influence the risk of NHL. The long-term effect of blood donation on cancer incidence has not been thoroughly investigated despite its potentially large impact on the population. A large nested case-control study within a cohort of blood donors in Sweden and Denmark revealed an increased risk of NHL among frequent plasma donors, but not among whole blood donors [9]. The few other epidemiological studies that evaluated the association between blood donation history and lymphoma risk showed conflicting results [10-13], and none estimated a dose-response relationship. Results of these studies have been difficult to interpret because of the possible influence of healthy donor effects or different baseline characteristics among blood donors compared to the respective reference population. Finally, none evaluated possible associations with specific NHL histologic subtypes, which may be etiologically distinct [14].

To test the hypothesis that frequent blood donation is associated with an increased risk of NHL and its most common histologic subtypes, we conducted a prospective study in the Health Professional Follow-up Study (HPFS) cohort.

\section{MATERIALS AND METHODS}

\section{Study Population}

The HPFS is a cohort study established in 1986. A total of 51,529 men between ages 40 to 75 years participated in the study. Every two years since its inception, participants have been mailed a self-administered questionnaire about demographic factors, lifestyle, and medical history. Information on blood donation history was collected for the first time in 1992 and was updated once in 1996. Thus, we defined the baseline population for the present analysis among the participants who returned the 1992 questionnaire $(n=46,203)$. From those, we excluded men who had an unknown date of NHL diagnosis $(n=13)$, had a history of cancer (except for non-melanoma skin cancer) $(\mathrm{n}=3662)$, or were missing data on blood donation history in 1992 ( $\mathrm{n}=5952)$, which left a total of 36,576 men in the analytic cohort. Comparing men who did and those who did not answer the blood donation question, there were no substantial differences with respect to age, body mass index, height, physical activity, smoking history, and alcohol consumption (data not shown). Vital status was identified from the National Death Index, report from next-of-kin or the post office (i.e., when a questionnaire mailed to a deceased participant was returned).

This study was approved by the Human Subjects Committee of the Harvard School of Public Health. Informed consent was implied by return of the baseline questionnaire.

\section{Case Ascertainment}

The outcome is incident diagnosis of NHL, identified by follow-up questionnaires and confirmed by review of medical records and pathology reports (98\%), death information, or self-confirmed by participant. Cases were defined according to the International Classification of Diseases, Eighth Revision (ICD-8) codes, 200, 202, and 204.1. Major histologic subtypes of NHL were determined according to the World Health Organization 
(WHO) classification of lymphomas [15] based on morphology and immunophenotype information available in medical records and the pathology report and consistent with recent International Lymphoma Epidemiology (InterLymph) Consortium guidelines [16,17]. For diagnoses of chronic lymphocytic leukemia / small lymphocytic lymphoma (CLL/SLL) and follicular lymphoma (FL), immunophenotype information was not required for tissue diagnoses as it was considered that morphology alone can reliably diagnose these histology subtypes [15]

\section{Exposure Assessment}

The exposure is self-reported history of blood donation as characterized by response to the 1992 and 1996 questionnaires. The 1992 questionnaire queried the total number of blood donations over the prior 30 years, with 8 response categories: never, 1-5, 6-9, 10-19, 20-29, $30-59,60-89$, or 90 donations or more. We collapsed the four highest categories (20-29, $30-59,60-89$, and 90 donations or more) into one category (i.e., 20+) to optimize statistical balance and stability. On the 1996 questionnaire, participants were asked to report total number of blood donations over the prior 4 years, with 8 response categories; never, 1-2, 3$4,5-8,9-12,13-16,17-20$, or 21 donations or more. We dichotomized this variable into never vs. 1 because relatively few men reported donating blood during this period.

\section{Statistical Analyses}

Person-time accrued for each participant from January 1992 (i.e., the mailing date of the 1992 questionnaire) to the earliest among dates of NHL diagnosis, death, or January 2010 (end of follow-up). Participants who died or reported cancer during follow-up were censored and excluded from subsequent follow-up. Cox proportional hazards models, stratifying jointly by calendar year of the current questionnaire cycle and age in months (i.e., the time scale), were used to estimate hazard ratios (HR) and 95\% confidence intervals (CI) for the risk of all NHL associated with number of blood donations $(1-5,6-9,10-19$, or $20+$, vs. none). Separate models were fit for the most common NHL histologic subtypes: diffuse large B-cell lymphoma (DLBCL), FL, and CLL/SLL. To evaluate potential confounding by putative NHL risk factors, we fit multivariable models that included the following covariates: smoking (never, past, or current), alcohol consumption (<0.5, 0.5-4.9, 5.0-9.9, 10.0-19.9, 20+ grams/day), body mass index (BMI; <23, 23-24.9, 25-26.9, 27-29.9, 30+ $\mathrm{kg} / \mathrm{m}^{2}$ ), physical activity (quintiles of metabolic equivalent (MET)-hours/week), height (centimeters, continuous), and race (White or other vs. Black or Asian) [18-22]. Covariates were updated with each questionnaire cycle and considered to be confounders if their inclusion in a given model resulted in substantial (i.e., >10\%) changes in the corresponding HR. To account for missing values for categorical covariates, we included a missing indicator category. Multivariable models for NHL histologic subtypes are not reported because of the small number of incident diagnoses of any given histologic subtype. To test our assumption of a linear trend between blood donation frequency and incidence of NHL, we fit a model using the mid-point of each blood donation category as a continuous variable. We used a contrast test, which followed an approximate $\chi^{2}$ distribution, to test whether associations of blood donation with major NHL histologic subtypes differed significantly $[23,24]$. Departures from the proportional hazards assumption, i.e. effect modification by 
age, was tested by likelihood ratio tests comparing models with and without the interaction terms of age by exposure.

We performed a separate analysis to evaluate associations between frequency of blood donation within 4 years and risk of NHL based on the 1996 questionnaire. We counted person-time from January 1996 (i.e. the mailing date of the 1996 questionnaire), and fit Cox proportional hazards models, stratifying by questionnaire cycle and age in months. Multivariable models were not fit given a relatively small case count in this reduced data set and the lack of evidence of substantial confounding in our main analysis. People with undiagnosed NHL may have symptoms that decrease their ability or willingness to donate blood. To diminish any possible influence of subclinical NHL on blood donation, a sensitivity analysis was performed, restricting analysis to person-time and NHL diagnoses that occurred from 1998 onward. We chose 2 years as the length of exposure lag in this sensitivity analysis because prior literature showed that donation intensity started to decrease no more than 2 years before the diagnosis of NHL [9].

All statistical tests were two-sided, and p-values $<0.05$ were considered statistically significant. We calculated a minimum detectable hazard ratio of 1.5 comparing extreme exposure categories and assuming 80\% power and an alpha level of 0.05 [25]. Analyses were performed with SAS Version 9 for UNIX (SAS Institute Inc.).

\section{RESULTS}

During 526,119 person-years of follow-up, 544 incident cases of NHL were confirmed, including 59 DLBCL, 55 FL, 189 CLL/SLL, 21 T-cell lymphoma, and 220 uncommon or unspecified B-cell lymphoma or unclassifiable for histologic subtype. Participant characteristics were not substantially different at baseline among blood donors and nondonors with respect to age, race, and distribution of potential NHL risk factors (Table 1).

Table 2 shows association between number of blood donations over a 30-year period and incidence of NHL. In the age-adjusted model, there was no significant association between blood donation frequency and incidence of NHL overall or of any histologic subtype evaluated (i.e., DLBCL, FL, or CLL/SLL) (Table 2). For example, the age-adjusted HR of all NHL comparing men in the highest blood donation category (i.e. 20+ over 30 years) with non-donors was 1.26 (95\% CI 0.94-1.68, p for trend $=0.18$ ). In the multivariable model, there was no evidence of confounding of the association between the incidence of all NHL and blood donation by covariates considered, including smoking history, alcohol consumption, body mass index, height, physical activity, and race (Table 2). Although inclusion of these selected covariates in multivariable models did not result in substantial differences in the HR estimates, we presented the multivariable model to allow comparison with previous literature. Given limited statistical power and lack of evidence of confounding, multivariable models are not presented for the analyses by major NHL histologic subtypes. There was no evidence of heterogeneity in effects by histologic subtypes of NHL ( $\mathrm{p}=0.86$ ). 
Similarly, there was no association between more recent blood donation history (i.e., within 4 years) and NHL in the analyses from 1996-2010 or after incorporating the 2-year exposure lag (data not shown).

\section{DISCUSSION}

In this large prospective cohort study, we found no evidence of an association between the frequency of blood donations and incidence rate of NHL or its major histologic subtypes.

Among five published articles about blood donation history and lymphoma [9-13] only one study assessed the dose-response relationship between blood donation frequency and lymphoma [9]. In this large nested case-control study in Swedish and Danish blood donors with 482 NHL cases, no significant association between blood donation overall and NHL was observed (OR 1.27, 95\% CI 0.84-1.94, comparing the highest ( $>25$ donations) vs. the lowest category of blood donations 3-12 years before diagnosis (1-8 donations), $\mathrm{P}_{\text {trend }}=$ 0.07). However, in analyses specific to blood donation types (i.e. whole blood donation or plasma donation), a significant positive association with NHL was noted for frequent plasma donors (OR 2.14, 95\% CI 1.22-3.74, comparing the highest ( $>25$ donations) vs. the lowest category of plasma donations $3-12$ years before diagnosis ( 0 donations), $\left.\mathrm{P}_{\text {trend }}=0.05\right)$. The authors acknowledged that this could be a chance finding in their setting of multiple comparisons and a limited number of cases, and that differences of socioeconomic status among frequent compared to less frequent plasma donors or potential unmeasured confounders may partially explain the plasma donor-specific finding [9]. In the present analysis, our study population of licensed health professionals is not likely to vary markedly by socioeconomic status, and we did not observe any evidence of confounding by putative risk factors such as alcohol consumption, smoking history, BMI and physical activity.

Three other studies, one of which overlapped with the Scandanavian study described above [9], reported no increase in the incidence of NHL among blood donors compared to the general population [10-12]. However, these null results may reflect the healthy lifestyle and other factors common among blood donors compared to general population [26]. In support of a healthy donor effect, a recent cohort study of blood donors in the Retroviral Epidemiology Donor Study (1991-2002) at the Blood Centers of the Pacific (BCP), San Francisco, who were linked to the California Cancer Registry reported an increased incidence of all cancers and of lymphoma (not further categorized) compared to the general U.S. population (SIR 1.72, 95\% CI 1.39-2.12) but significantly lower overall mortality among donor cancer patients [13]. The authors speculated that the observed increase in cancer incidence among blood donors was due to the differences in other sociodemographic characteristics, such as age, race, and education, as well as differences in health-seeking behavior and access to health care, rather than a true etiologic association.

No previous studies have reported possible associations of blood donation and histologic subtypes of NHL, which may be etiologically distinct [14]. In our subtype analyses, which were exploratory in nature, we did not observe an association between blood donation and any major subtype, and there was no evidence of heterogeneity in effects by subtypes. These results should be interpreted with caution because of limited statistical power. 
Severe immune deficiency is well established to increase the risk of NHL [15].

Accumulating evidence shows that not only immune deficiency but also conditions that trigger chronic immune stimulation, such as autoimmune diseases and certain infections have etiologic roles in NHL [3,27-29]. Blood donation could influence subsequent NHL development via temporary immune system alterations following blood donation, which have been demonstrated in some [6-8], but not all [30], human studies. Studies that specifically investigated the influence of plasma donation on the immune system have also shown conflicting results [31-33]. It is difficult to speculate whether immune system alterations after blood donation are truly transient or substantial enough to cause observable long-term health effects.

There are important limitations to our analyses. First, blood donation history was queried only twice during cohort follow-up, and we did not have information on the timing or type of reported blood donations (e.g., plasma vs. whole blood). We therefore could not investigate how the timing and patterns of blood donations may have influenced the results. Second, the information on blood donation frequency was obtained through self-reported questionnaire and was not confirmed objectively, which could lead to exposure misclassification. We identified a single report suggesting that while there is high agreement between self-reported blood donation and record-confirmed donation for ever vs. never, study participants over-reported frequency of donation [34]. However, in a subset of 305 HPFS participants with serum ferritin measures (a marker of iron stores), the mean ferritin levels were about 2 -fold lower in the high donation groups (e.g. 30+ lifetime donations) compared to non-donors, indicating that self-report has been well validated in this population [35]. Further, given the prospective design of our study, exposure misclassification is generally expected to be non-differential with respect to NHL diagnosis, but it might have led to an attenuation of a weak association if present. Moreover, we had a relatively limited number of incident cases and therefore low statistical power, particularly for subtype analysis, thus we cannot exclude the possibility of a weak association. No exclusions were made for HIV status, other viral or bacterial infections, or organ transplantation, endpoints that have not been formally assessed in follow-up. These factors could be related to both blood donation and NHL risk; however, the prevalence of these conditions is expected to be rare and therefore any bias due to confounding by these factors is likely to be minimal. While follow-up rates in the HPFS are typically $>90 \%$, some incident NHL cases that did not result in death could have been missed due to loss to followup. Lastly, our study was based in a male-only cohort, and because it is not known whether there are differences in temporary immune system activity by sex, we cannot make an inference about a possible blood donation- NHL association among females.

Our study has several strengths that merit consideration, including its prospective design, high follow-up rate, long follow-up duration, and large cohort size. While previous studies have been limited by the potential influence of a possible "healthy donor effect," the HPFS is comprised entirely of U.S. health professionals who are expected to be of similar socioeconomic status and have similar levels of access to care. Moreover, detailed highquality information on lifestyle factors allowed us to evaluate potential confounding by putative NHL risk factors, although we cannot exclude the possibility of residual confounding by unmeasured factors that may correlate with frequency of blood donation. 
While it is reassuring that our large prospective cohort study did not show evidence of an association between blood donation frequency and incidence of NHL, further exploration of the magnitude and temporal patterns of immune system alterations after blood donation and future epidemiological studies might provide new insights about blood donation safety, and subsequently about lymphomagenesis.

\section{Acknowledgments}

The authors thank the participants and staff of the HPFS for their valuable contributions as well as the following state cancer registries for their help: AL, AZ, AR, CA, CO, CT, DE, FL, GA, ID, IL, IN, IA, KY, LA, ME, MD, MA, MI, NE, NH, NJ, NY, NC, ND, OH, OK, OR, PA, RI, SC, TN, TX, VA, WA, and WY. The authors assume full responsibility for analyses and interpretation of these data. This work was supported by the NIH (CA055075, CA167552 and CA149445) and the American Cancer Society (RSG-11-020-01-CNE). K.A. Bertrand was supported by the Simeon J. Fortin Charitable Foundation, Bank of America, N.A., Co-Trustee.

\section{References}

1. Muller AM, Ihorst G, Mertelsmann R, Engelhardt M. Epidemiology of non-Hodgkin's lymphoma (NHL): trends, geographic distribution, and etiology. Ann Hematol. 2005; 84:1-12. [PubMed: 15480663]

2. Alexander DD, Mink PJ, Adami HO, et al. The non-Hodgkin lymphomas: a review of the epidemiologic literature. Int J Cancer. 2007; 120(Suppl 12):1-39. [PubMed: 17405121]

3. Grulich AE, Vajdic CM, Cozen W. Altered immunity as a risk factor for non-Hodgkin lymphoma. Cancer Epidemiol Biomarkers Prev. 2007; 16:405-408. [PubMed: 17337643]

4. Lan Q, Zhang L, Tang X, et al. Occupational exposure to trichloroethylene is associated with a decline in lymphocyte subsets and soluble CD27 and CD30 markers. Carcinogenesis. 2010; 31:1592-1596. [PubMed: 20530238]

5. Saberi Hosnijeh F, Krop EJ, Scoccianti C, et al. Plasma cytokines and future risk of non-Hodgkin lymphoma (NHL): a case-control study nested in the Italian European Prospective Investigation into Cancer and Nutrition Cancer. Epidemiol Biomarkers Prev. 2010; 19:1577-1584.

6. Marquet RL, Hoynck van Papendrecht MA, Busch OR, Jeekel J. Blood donation leads to a decrease in natural killer cell activity: a study in normal blood donors and cancer patients. Transfusion. 1993; 33:368-373. [PubMed: 8488538]

7. Karger R, Weber C, Schmidt J, Kretschmer V. Characterization of immune system alterations following preoperative autologous blood donation for elective hip replacement surgery. Transfus Med. 2007; 17:45-53. [PubMed: 17266703]

8. Lasek W, Plodziszewska M, Jakobisiak M. The effect of blood donation on natural killer activity in man. J Clin Lab Immunol. 1987; 22:165-168. [PubMed: 3612753]

9. Edgren G, Reilly M, Hjalgrim H, et al. Donation frequency, iron loss, and risk of cancer among blood donors. J Natl Cancer Inst. 2008; 100:572-579. [PubMed: 18398098]

10. Edgren G, Tran TN, Hjalgrim H, et al. Improving health profile of blood donors as a consequence of transfusion safety efforts. Transfusion. 2007; 47:2017-2024. [PubMed: 17958530]

11. Merk K, Mattsson B, Mattsson A, Holm G, Gullbring B, Bjorkholm M. The incidence of cancer among blood donors. Int J Epidemiol. 1990; 19:505-509. [PubMed: 2262240]

12. Lasek W, Jakobisiak M, Stoklosa T. Decreased natural killer cell activity in whole-blood donors does not seem to result in increased cancer incidence. Transfusion. 1994; 34:359-360. [PubMed: 8178337]

13. Vahidnia F, Hirschler NV, Agapova M, Chinn A, Busch MP, Custer B. Cancer Incidence and Mortality in a Cohort of US Blood Donors: A 20-Year Study. J Cancer Epidemiol. 2013; 2013:814842. [PubMed: 24489545]

14. Morton LM, Wang SS, Cozen W, et al. Etiologic heterogeneity among non-Hodgkin lymphoma subtypes. Blood. 2008; 112:5150-5160. [PubMed: 18796628] 
15. Swerdlow, SH.; Campo, E.; Harris, NL., et al., editors. WHO Classification of Tumours of Haematopoietic and Lymphoid Tissues. Lyon, France: International Agency for Research on Cancer (IARC) Press; 2008.

16. Morton LM, Turner JJ, Cerhan JR, et al. Proposed classification of lymphoid neoplasms for epidemiologic research from the Pathology Working Group of the International Lymphoma Epidemiology Consortium (InterLymph). Blood. 2007; 110:695-708. [PubMed: 17389762]

17. Turner JJ, Morton LM, Linet MS, et al. InterLymph hierarchical classification of lymphoid neoplasms for epidemiologic research based on the WHO classification (2008): update and future directions. Blood. 2010; 116:e90-98. [PubMed: 20699439]

18. Diver WR, Patel AV, Thun MJ, Teras LR, Gapstur SM. The association between cigarette smoking and non-Hodgkin lymphoid neoplasms in a large US cohort study. Cancer Causes Control. 2012; 23:1231-1240. [PubMed: 22689321]

19. Bertrand KA, Giovannucci E, Zhang SM, Laden F, Rosner B, Birmann BM. A prospective analysis of body size during childhood, adolescence, and adulthood and risk of non-Hodgkin lymphoma. Cancer Prev Res (Phila). 2013; 6:864-873. [PubMed: 23803416]

20. Kelly JL, Fredericksen ZS, Liebow M, et al. The association between early life and adult body mass index and physical activity with risk of non-Hodgkin lymphoma: impact of gender. Ann Epidemiol. 2012; 22:855-862. [PubMed: 23146413]

21. Morton LM, Zheng T, Holford TR, et al. Alcohol consumption and risk of non-Hodgkin lymphoma: a pooled analysis. Lancet Oncol. 2005; 6:469-476. [PubMed: 15992695]

22. Cancer incidence in five continents. Volume IX. IARC Sci Publ. 2008:1-837.

23. Smith-Warner SA, Spiegelman D, Ritz J, et al. Methods for pooling results of epidemiologic studies: the Pooling Project of Prospective Studies of Diet and Cancer. Am J Epidemiol. 2006; 163:1053-1064. [PubMed: 16624970]

24. Anderson, TW. Introduction to Multivariate Statistics. New York: John Wiley \& Sons; 1984.

25. Chapman DG, Nam JM. Asymptotic power of chi square tests for linear trends in proportions. Biometrics. 1968; 24:315-327. [PubMed: 5683872]

26. Atsma F, Veldhuizen I, Verbeek A, de Kort W, de Vegt F. Healthy donor effect: its magnitude in health research among blood donors. Transfusion. 2011; 51:1820-1828. [PubMed: 21342203]

27. Ekstrom Smedby K, Vajdic CM, Falster M, et al. Autoimmune disorders and risk of non-Hodgkin lymphoma subtypes: a pooled analysis within the InterLymph Consortium. Blood. 2008; 111:4029-4038. [PubMed: 18263783]

28. Edlefsen KL, Martinez-Maza O, Madeleine MM, et al. Cytokines in serum in relation to future non-Hodgkin lymphoma risk: Evidence for associations by histologic subtype. Int J Cancer. 2014

29. Vendrame E, Hussain SK, Breen EC, et al. Serum levels of cytokines and biomarkers for inflammation and immune activation, and HIV-associated non-Hodgkin B-cell lymphoma risk. Cancer Epidemiol Biomarkers Prev. 2014; 23:343-349. [PubMed: 24220912]

30. Lewis SL, Kutvirt SG, Simon TL. Investigation of the effect of long-term whole blood donation on immunologic parameters. Transfusion. 1992; 32:51-56. [PubMed: 1731436]

31. Lewis SL, Kutvirt SG, Bonner PN, Simon TL. Plasma proteins and lymphocyte phenotypes in long-term plasma donors. Transfusion. 1994; 34:578-585. [PubMed: 8053039]

32. Tran-Mi B, Storch H, Seidel K, et al. The impact of different intensities of regular donor plasmapheresis on humoral and cellular immunity, red cell and iron metabolism, and cardiovascular risk markers. Vox Sang. 2004; 86:189-197. [PubMed: 15078254]

33. Schulzki T, Seidel K, Storch H, et al. A prospective multicentre study on the safety of long-term intensive plasmapheresis in donors (SIPLA). Vox Sang. 2006; 91:162-173. [PubMed: 16907878]

34. Bertalli NA, Allen KJ, McLaren CE, et al. A comparison of self-reported and record-linked blood donation history in an Australian cohort. Transfusion. 2011; 51:2189-2198. [PubMed: 21985049]

35. Zhang X, Ma J, Wu K, Chan AT, Fuchs CS, Giovannucci EL. Blood donation and colorectal cancer incidence and mortality in men. PLoS One. 2012; 7:e39319. [PubMed: 22761761] 

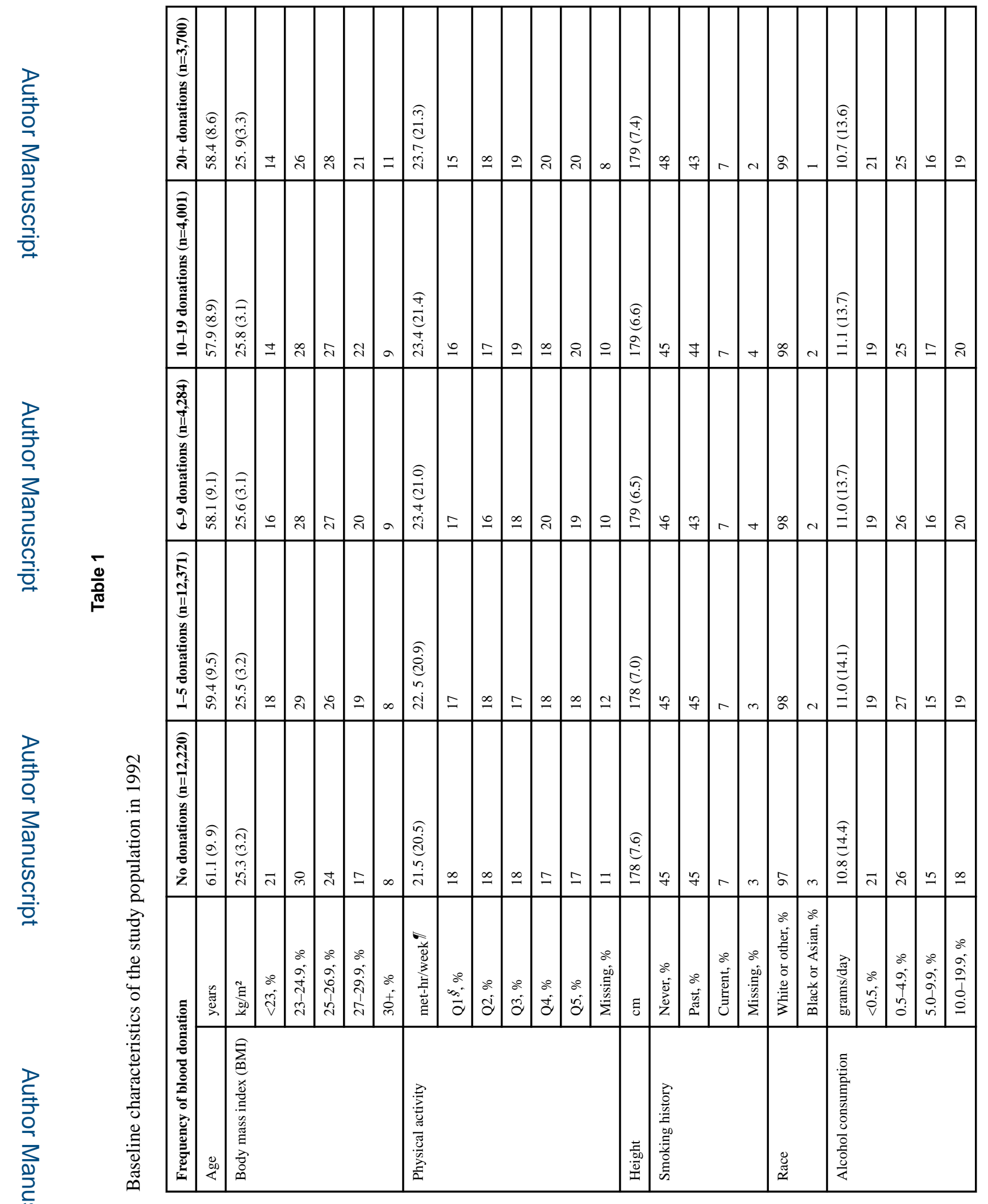

Leuk Lymphoma. Author manuscript; available in PMC 2017 June 01. 


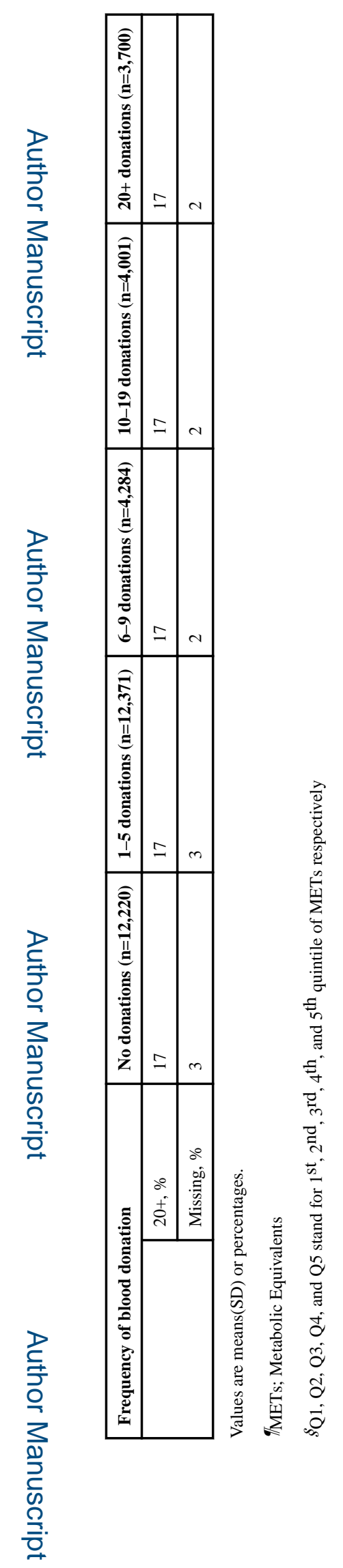




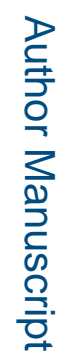

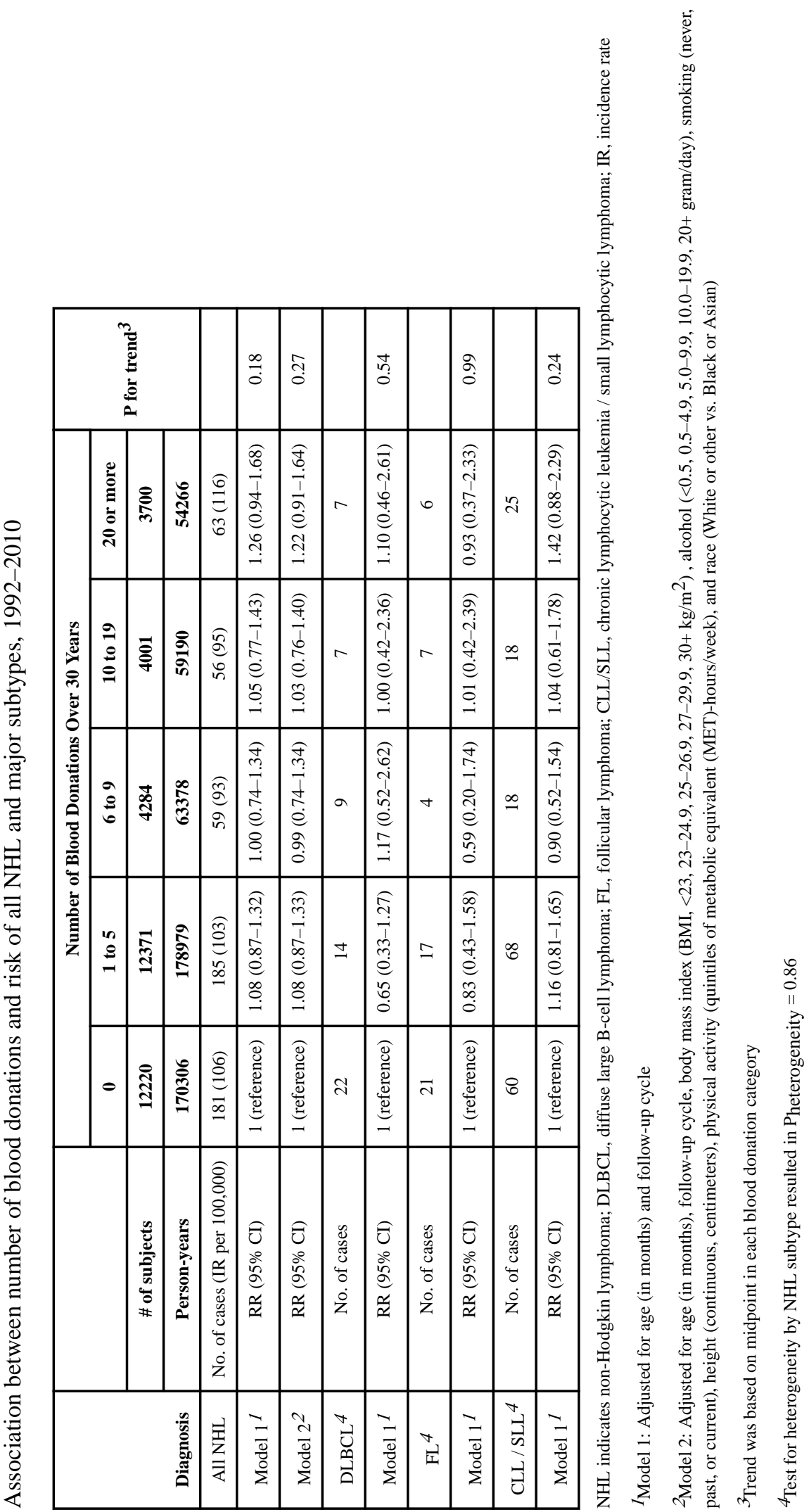

Leuk Lymphoma. Author manuscript; available in PMC 2017 June 01. 